\title{
A Novel Microcontroller-Based System for the Wheel-Running Activity in Mice
}

\author{
DMeina Zhu, ${ }^{1}$ Deepa Kamath Kasaragod, ${ }^{1}$ Kazuya Kikutani, ${ }^{2}$ 'Kei Taguchi, ${ }^{1}$ and Hidenori Aizawa ${ }^{1}$
}

\author{
https://doi.org/10.1523/ENEURO.0260-21.2021
}

${ }^{1}$ Department of Neurobiology, Graduate School of Biomedical and Health Sciences, Hiroshima University, Hiroshima 734-8553, Japan and ${ }^{2}$ Department of Emergency and Critical Care Medicine, Graduate School of Biomedical and Health Sciences, Hiroshima University, Hiroshima 734-8553, Japan

\begin{abstract}
Voluntary wheel-running activity is a way to assess rodents' circadian rhythm and motivation for exercise. Deficits in these behaviors are implicated in the pathophysiology of sleep and psychiatric disorders. Limited space in animal facilities can hamper long-term monitoring of running wheel activity outside of the home cage. To address this issue, we provide a stand-alone solution to monitor the wheel-running activity of mice in their home cage. This system, named the wheel-running activity acquisition (WRAQ) system, is based on a microcontroller driven by a lithium polymer battery. With the WRAQ, we can record the wheel-running activity and illumination data for at least $30 \mathrm{~d}$. Applying the WRAQ to an endotoxemia mouse model robustly detected the altered wheel-running activity and its recovery. With wireless data transfer capability extension, the system also allows for online monitoring and reporting of the circadian time (CT). We used the online monitoring of wheel-running activity with this extended WRAQ system and observed a significant shift of the active period in the circadian rhythm following a temporal chemogenetic activation of the suprachiasmatic nucleus (SCN)-subparaventricular zone (SPZ). Together, these findings indicate that the WRAQ system is a novel and cost-effective solution for the analysis of wheel-running activity in mice.
\end{abstract}

Key words: wheel running; circadian rhythm; open-source; mouse; microcontroller

\section{Significance Statement}

Wheel-running activity is commonly used to assess voluntary activity along with the circadian rhythm in rodents. Long-term recording of the activity within additional animal facility space and associated costs could hamper its use depending on the scale of the study. Here, we provide a cost-effective and stand-alone solution to measure wheel-running activity in the home cage following manipulation of the central nervous system. We used a microcontroller for an Internet of things solution to monitor behavioral and environmental data online. This novel approach may ultimately contribute to the real-time analysis of rodent behaviors during temporal genetic and pharmacological interventions.

\section{Introduction}

Behavioral activity in the home cage is a basic phenotype analyzed in neuroscience animal studies. In particular, voluntary wheel-running activity changes are often associated with diseases in animal models (Siepka and Takahashi, 2005). For example, previous studies identified genes modulating the circadian rhythm of wheel-

Received June 8, 2021; accepted August 28, 2021; First published September 3, 2021.

The authors declare no competing financial interests. running activity by analyzing the activity of mutants (Takahashi, 2017). Despite requiring additional energy, access to a running wheel increases voluntary activity in most rodents, which might benefit phenotypic analyses by amplifying the differences in activity between control and mutant groups. This is especially the case when examining the circadian rhythm of wheel-running activity

Author contributions: H.A. designed research; M.Z., K.K., and K.T. performed research; H.A., M.Z., and D.K.K. analyzed data; H.A. and M.Z. wrote the paper. 
since voluntary activity is generally restricted to the active period of the circadian rhythm in rodents (Novak et al., 2012).

Equipment to measure wheel-running activity in mice is available commercially and mainly consists of a running wheel and a data acquisition system, which are placed inside and outside of the cage, respectively. Considering the high-density rack systems with smaller cages that house mice under specific pathogen-free conditions, equipment providing stand-alone operation and remote reporting of the acquired data online would be desirable for wheel-running activity analysis in mice. Streaming behavioral and environmental signals online would allow researchers to analyze the data in real time and manipulate the ongoing neural activity at specific circadian times (CTs) using genetic and pharmacological interventions.

We developed an open-source hardware system named wheel-running activity acquisition (WRAQ) based on a microcontroller recording mice's voluntary wheelrunning activity in their home cage. This system combines a low-profile running wheel with a reed switch and photoresistor for data acquisition, operating with a lithium polymer battery for at least $30 \mathrm{~d}$ and storing data on a microSD card for offline analysis. We validated the WRAQ system with a behavioral study by performing quantitative analysis of mice under different schedules of light entrainment and with systemic inflammation as a disease model. We further extended WRAQ to enable online monitoring of wheel-running activity using wireless recording capability. This capability allowed chemogenetic activation of specific neuronal pathways in a temporally specific manner.

\section{Materials and Methods}

\section{Animals}

All procedures involving animals were performed per the ARRIVE guidelines (https://arriveguidelines.org/arriveguidelines) and were approved by the institutional experimental animal committee (A18-42-2 and A16-46-2). C57BL/6J mice (seven to eight weeks old, male; CLEA) were housed individually in plastic cages (CL-0104-2, width $225 \times$ depth $338 \times$ height $140 \mathrm{~mm}$, CLEA) with free

This work was supported by the Ministry of Education, Culture, Sports, Science and Technology (MEXT) KAKENHI Grants JP19H05723, 21H02581, and 21H00203 and a Grant-in-Aid for "Integrated Research on Depression, Dementia and Development Disorders" (JP18dm0107093h0003) carried out under the Strategic Research Program for Brain Sciences by Japan Agency for Medical Research and Development (AMED). M.Z. is a recipient of Otsuka Toshimi scholarship.

Acknowledgements: We thank Saori Okamura, Fumie Nishimura, and the Natural Science Center for Basic Research and Development of Hiroshima University for the technical assistance and Enago for the English language review.

Correspondence should be addressed to Hidenori Aizawa at haizawa@ hiroshima-u.ac.jp.

https://doi.org/10.1523/ENEURO.0260-21.2021 Copyright $\odot 2021$ Zhu et al.

This is an open-access article distributed under the terms of the Creative Commons Attribution 4.0 International license, which permits unrestricted use, distribution and reproduction in any medium provided that the original work is properly attributed. access to food and water, a 12/12 h light/dark cycle (LD), constant darkness (DD), or constant light illumination (LL; 120 lux), and regulated temperature and humidity in the range of $18-25^{\circ} \mathrm{C}$ and $30-60 \%$, respectively. For the experiments conducted under DD or LL conditions, mice were housed individually three weeks before recording.

\section{Design of WRAQ and its extension with wireless data transfer capability (WRAQ-WiFi)}

WRAQ was built based on a low-profile running wheel (flying saucer exercise wheel for small pets 5 inches, Ware Manufacturing Inc., width 5 inches $\times$ depth 5 inches $\times$ height 3.5 inches; Fig. $1 A$, left). A microcontroller managed data acquisition with a microSD card writer, Adafruit Feather MO Adalogger, connected to a binary counter. The revolution of a small round magnet glued to the bottom of the wheel was detected by a reed switch attached to the main body of WRAQ (Fig. $1 B$ ). Upon sweeping the reed switch by the magnet, the number of revolutions was counted by the binary counter. Adalogger was in deep sleep mode to save power for long-term recording and woke up every $4 \mathrm{~s}$ to check the counter and voltage across the cadmium sulfide photoresistor (MI527, Macron International Group Ltd.) for illumination data. We stored the resultant data on a microSD card with the timestamps of the onboard realtime clock (Fig. 1A, middle). WRAQ was powered by either a lithium polymer battery (2000 mAh, Shenzhen Data Power Technology Ltd.) or a lithium AA battery (3.6V, Guangzhou Markyn Battery Co, Ltd.; Fig. 1C). The reed switch signal was connected to the binary counter through an $\mathrm{RC}$ lowpass filter to suppress chattering (Fig. 2A).

We extended WRAQ to WRAQ-WiFi, which enables online monitoring of ongoing wheel-running activity (Fig. 1D). The capability to upload data to the online data storage was implemented by WiFi connectivity using a built-in FireBeetle ESP32 IoT microcontroller (DFR0478, DFRobot), which replaced the Adalogger. In addition, we attached a real-time clock breakout board (catalog \#3013, Adafruit Industries or zs-042, HiLetgo) based on the real-time clock DS3231 (Maxim Integrated), allowing ESP32 to access time stamps via an $I^{2} \mathrm{C}$ protocol. We acquired the number of revolutions and the illumination data as in WRAQ. ESP32 was set to wake up from deep sleep mode and upload the data to the Ambient loT data visualization cloud service (AmbientData Inc., https:// ambidata.io/), enabling users to monitor the ongoing and collected data online (Fig. 1A, middle).

We inserted all the parts, except the magnet and reed switch, into the main body of the flying saucer, covered it with a 3D-printed plastic part, and sealed it with a peelable silicon adhesive (1690, Amon Industry Co, Ltd.).

\section{Analysis of the wheel-running activity data}

ActogramJ software (https://bene51.github.io/ActogramJ/; Schmid et al., 2011), which is based on ImageJ (http:// imagej.nih.gov/ij/; Schneider et al., 2012), was used for offline analysis of the wheel-running activity data retrieved from the 

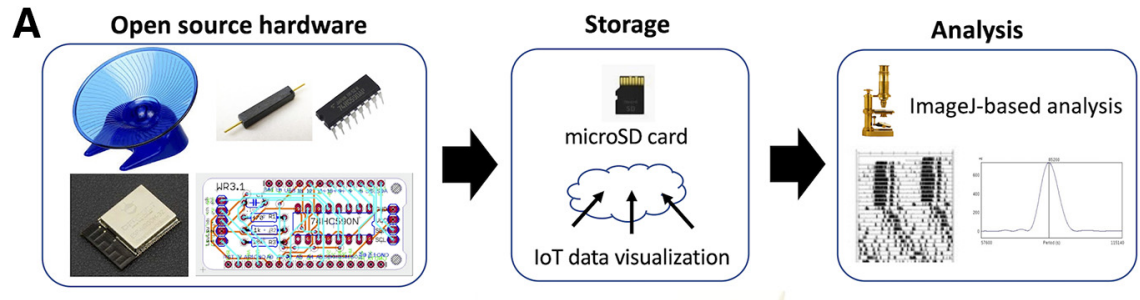

B

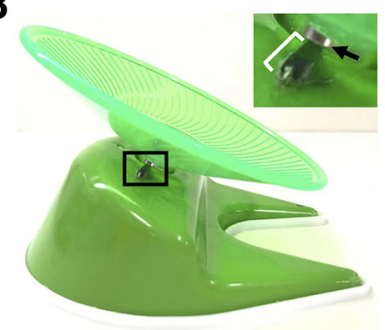

C

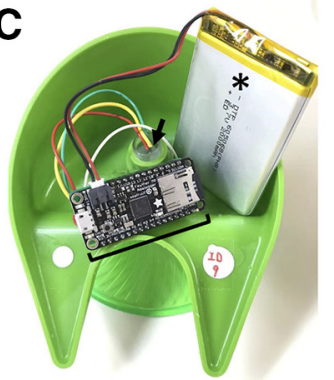

D

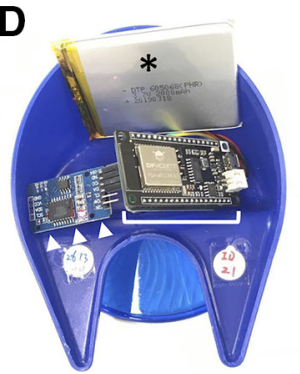

Figure 1. Overview of the WRAQ system. A, Schematic showing the WRAQ system workflow. The WRAQ system acquires data using a low-profile running wheel detecting the number of revolutions and light intensity in the cage using a magnet-reed switch and photodiode sensor, respectively (left). Data are stored either to a microSD card or an online server via WiFi connection (middle). Analyses with actogram and periodogram are conducted offline using a software-based on ImageJ (right). $\boldsymbol{B}-\boldsymbol{D}$, Side (B) or bottom views $(\boldsymbol{C}, \boldsymbol{D})$ of the WRAQ $(\boldsymbol{B}, \boldsymbol{C})$ and WRAQ-WiFi $(\boldsymbol{D})$ showing the reed switch (bracket in $\boldsymbol{B})$ on the main body detecting the sweep by a magnet attached to the bottom of the rotating wheel (arrow in $\boldsymbol{B}$ ) and microcontrollers (brackets). The WRAQ and WRAQ-WiFi microcontrollers, i.e., Adalogger (bracket in $\mathbf{C}$ ) and FireBeetle ESP32 (bracket in $\boldsymbol{D}$ ), are connected to a lithium polymer battery (asterisks). Note that the photoresistor is encased in a silicon tube and placed at the center of the main body (arrow in $\boldsymbol{C}$ ). Inset shows a magnified view of a boxed area in $\boldsymbol{B}$. White arrowheads, real-time clock module.

microSD card (WRAQ) or downloaded from the cloud service (WRAQ-WiFi; Fig. 1A, right). The raw data were converted into the file readable by ActogramJ, in which the data were supposed to start at zeitgeber time (ZT)12, by custom-made python program (main_wraq2actj.py provided as Extended Data). The actogram and periodogram using Lomb-Scargle methods were calculated as described previously (Schmid et al., 2011).

\section{Treatment with lipopolysaccharide (LPS)}

Three weeks after habituation under DD, mice were administered a single intraperitoneal injection of saline or LPS derived from Escherichia coli (O55:B5, L2880, Sigma-Aldrich) at a dose of $2.5 \mathrm{mg} / \mathrm{kg}$. Their wheel-running activity was continuously recorded with the WRAQ system placed in their home cage across the LPS injection.

\section{Chemogenetic activation of the suprachiasmatic nucleus (SCN)-subparaventricular zone (SPZ)}

We anesthetized mice with a mixture of ketamine $(90 \mathrm{mg} / \mathrm{kg})$ and xylazine $(10 \mathrm{mg} / \mathrm{kg})$ and immobilized it in a stereotaxic frame (SR6N, Narishige). Following a midline incision of the skin covering the skull, we made a burrhole to open a cranial window at $0.48 \mathrm{~mm}$ posterior and 0.15 $\mathrm{mm}$ lateral to the bregma over the SCN-SPZ (Franklin and Paxinos, 2008). A fine glass capillary was used to inject $0.05 \mu \mathrm{l}$ of AAV8-hSyn-hM3D(Gq)-mCherry $(2.5 \times 10 \mathrm{e} 12$ $\mathrm{gc} / \mathrm{ml}$, catalog \#50474, Addgene; RRID:Addgene_50474) or AAV8-CAG-GFP $(2 \times 10 \mathrm{e} 12 \mathrm{vm} / \mathrm{ml}$, UNC GTC Vector Core, University of North Carolina, Chapel Hill, NC) with a speed of $0.1 \mu \mathrm{l} / \mathrm{min}$ targeting the bilateral SCN-SPZ (5.8 and $5.7 \mathrm{~mm}$ deep from the pia mater). After closing the skin covering the cranial window by suture, the mouse was allowed to recover in its home cage for at least $7 \mathrm{~d}$. Then, we administered a solution of clozapine $\mathrm{N}$-oxide (CNO; $1 \mathrm{mg} / \mathrm{kg}$, BML-NS105-0005, Enzo Life Sciences) by intraperitoneal injection to activate cells expressing hM3D.

\section{Data analysis}

We converted illumination data recorded from the WRAQ and WRAQ-WiFi systems into a $z$ score using each mean and standard deviation. The wheel revolutions were represented using 4-s (WRAQ) or 64-s (WRAQ-WiFi) bins. We discarded part of the raw data so it would start at ZT12 and then imported into ActogramJ to calculate a periodogram using the Lomb-Scargle method. Presumptive distance traveled was calculated by multiplying the number of wheel revolutions recorded by WRAQ or WRAQWiFi with the perimeter of the presumptive trace on the running wheel $(25.12 \mathrm{~cm})$. For analysis using periodogram, we excluded data with wheel-running activity under 20,000 revolutions per day.

\section{Immunohistochemistry}

Two hours after intraperitoneal injection of $\mathrm{CNO}$, mice with AAV8-hSyn-hM3D(Gq)-mCherry or AAV8-CAG-GFP were perfused transcardially using $4 \%$ paraformaldehyde (PFA) in $0.1 \mathrm{~m}$ PBS. After that, we dissected the brain and postfixed it in the same fixative overnight at $4^{\circ} \mathrm{C}$. Then, $75-\mu \mathrm{m}$-thick coronal sections were cut using a vibratome 
A

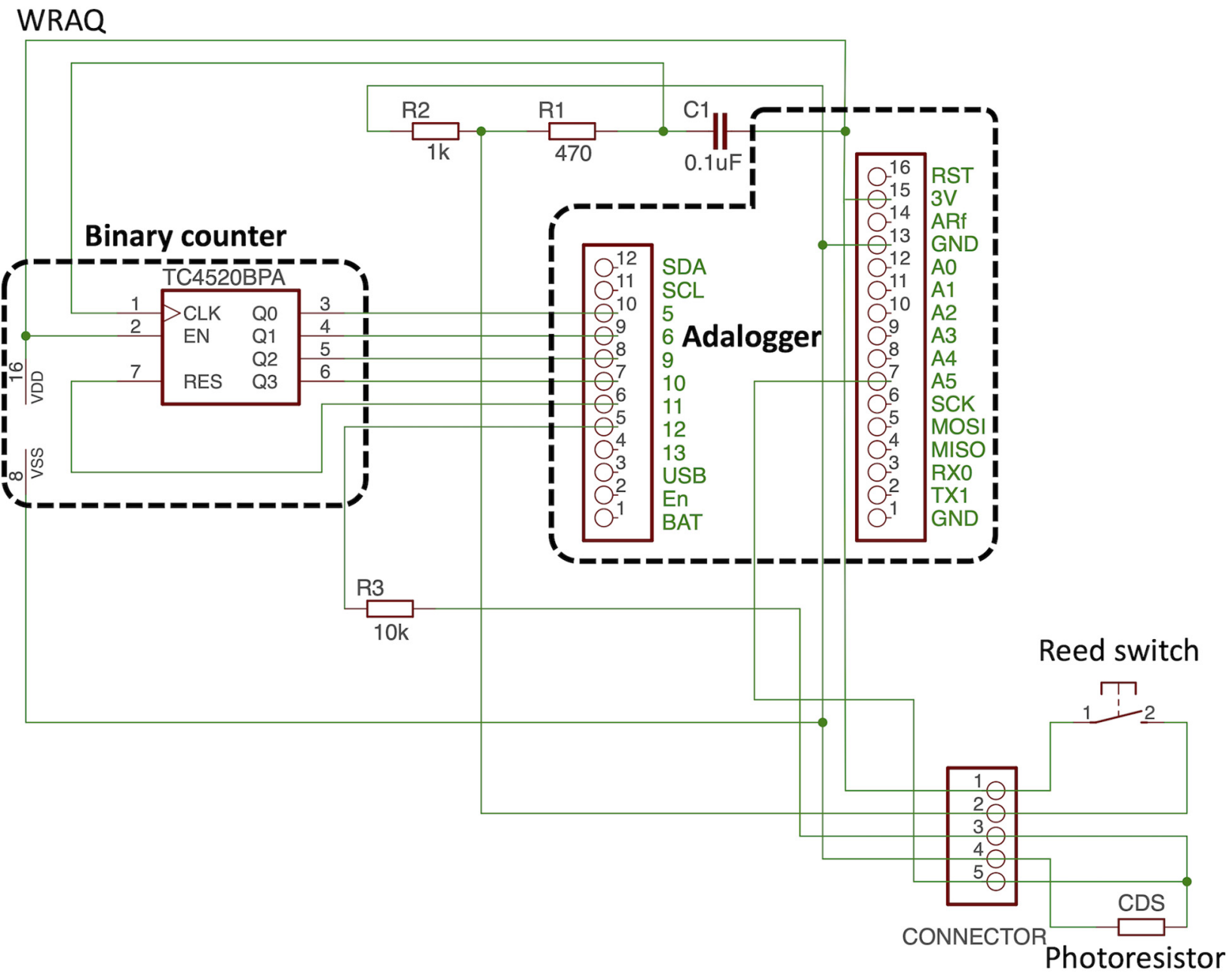

B

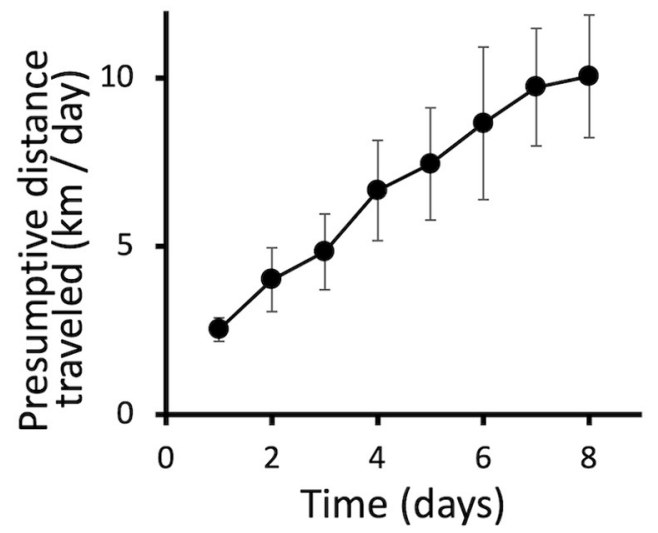

C

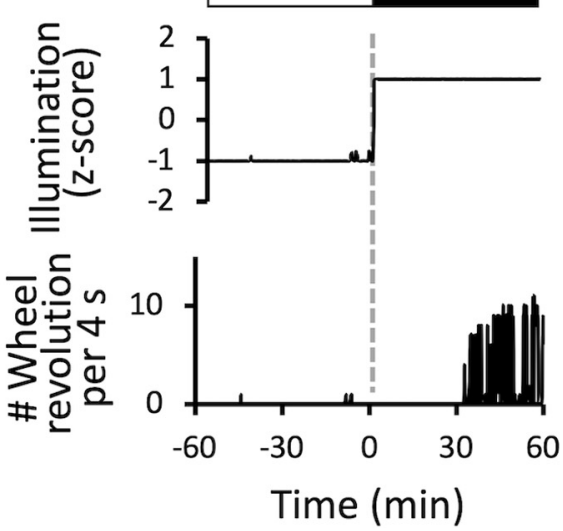

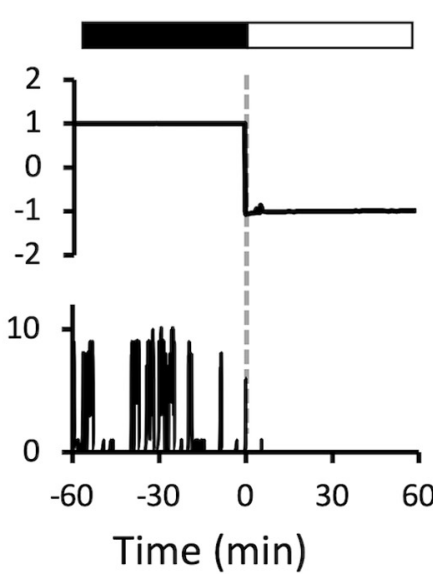

Figure 2. Simultaneous acquisition of the wheel revolution number and illumination data under light-dark light entrainment. $\boldsymbol{A}$, A schematic showing the hardware part of the WRAQ system primarily consists of a microcontroller for recording and system management (Adalogger) and a 4-bit binary counter which counts the number of wheel revolutions when the Adalogger is in deep sleep mode. $\boldsymbol{B}$, Line plot of the daily wheel-running activity during habituation to the WRAQ system. Values are represented as mean \pm SEM. C, Temporal changes of the normalized illumination (voltage across the photoresistor, upper traces) and the number of wheel revolutions per $4 \mathrm{~s}$ (bottom trances) are shown across the transitions between light and dark period (dashed gray lines). $\mathrm{A}$, analog input; C, capacitor; CDS, cadmium sulfide photoresistor; CLK, clock input; EN, enabled; GND, ground; R, resistor; RES, reset; VDD, voltage drain; VSS, voltage source. 

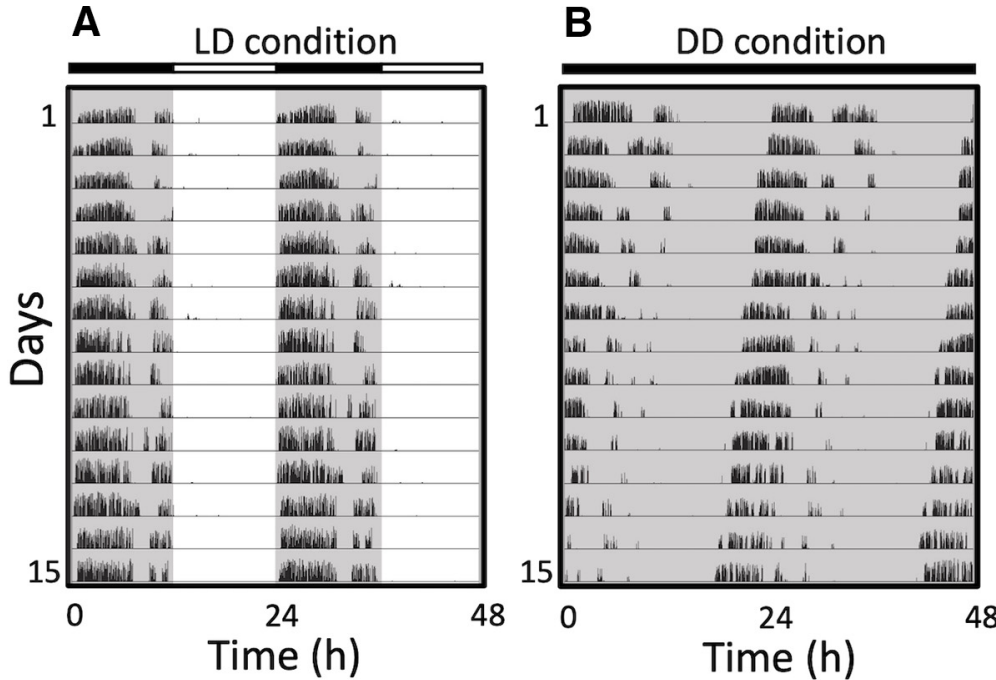

E

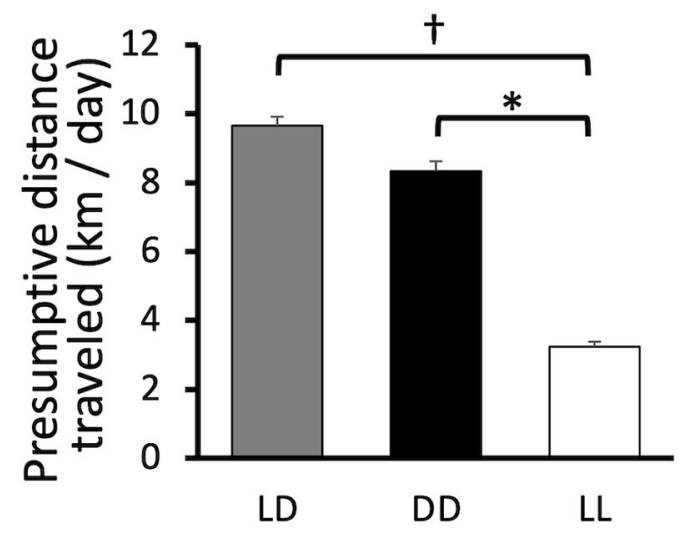

\section{C}

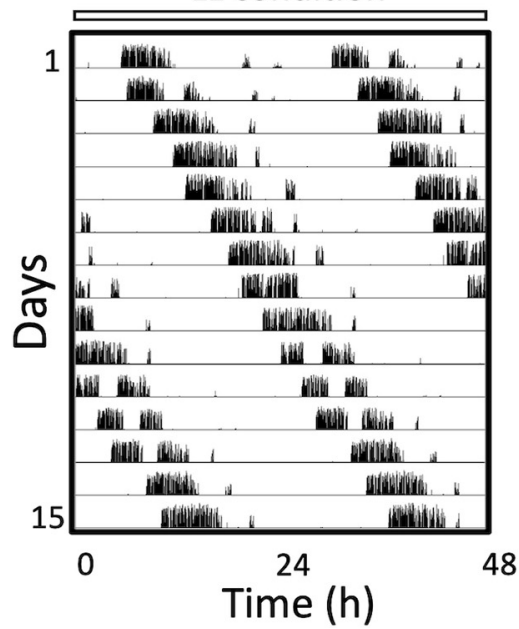

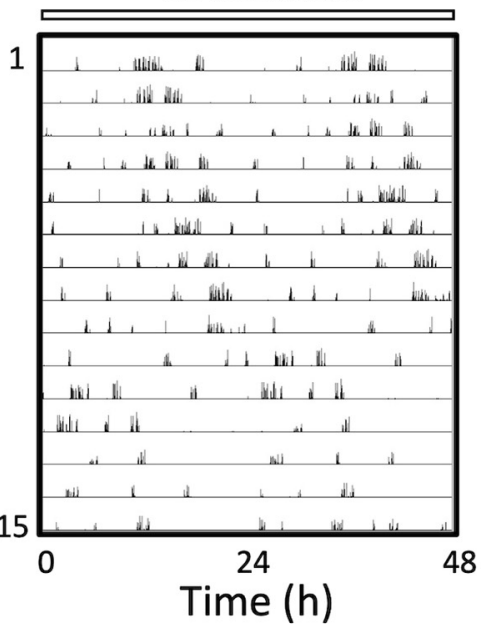

\section{LL condition}

$\mathbf{F}$

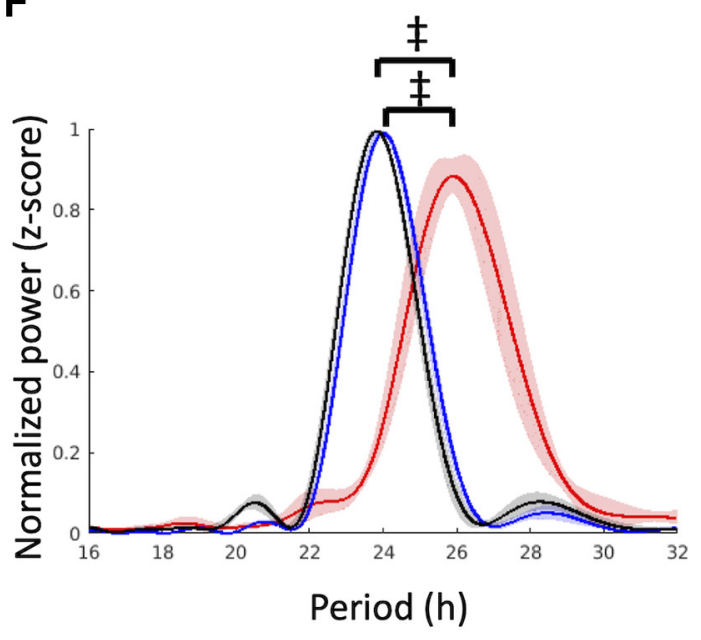

Figure 3. Wheel-running activity acquired by the WRAQ system under different entrainment schedules. $\boldsymbol{A}-\boldsymbol{D}$, Actograms of mouse wheel-running activity under LD $(\boldsymbol{A})$, DD $(\boldsymbol{B})$, and $\operatorname{LL}(\boldsymbol{C}, \boldsymbol{D})$ conditions. $\boldsymbol{E}$, Bar graph of the presumptive distance traveled on the wheel for mice kept under LD (gray), DD (black), and LL (white) entrainment. $\boldsymbol{F}$, Line plots of the periodograms analyzing wheel-running activity recorded for $8 \mathrm{~d}$ under LD (blue, $n=10$ ), DD (gray, $n=10$ ), and LL (red, $n=7$ ) based on the Lomb-Scargle method. Data are presented as mean (solid lines) \pm SEM (error bars and shading). Contrasts are statistically significant differences of mean values between groups (repeated-measures ANOVA followed by Tukey's post hoc test); ${ }^{*}<0.05, \uparrow p<0.01, \neq p<0.001$.

(DTK-1500, Dosaka EM Co, Ltd.) from 0.1 to $0.9 \mathrm{~mm}$ posterior to the bregma (Franklin and Paxinos, 2008). Sections were washed with $0.5 \%$ PBS Triton X-100 and incubated in primary antibody against c-Fos (1:500, sc271243, Santa Cruz Biotechnology) dissolved in 1\% blocking reagent in $0.5 \%$ PBS Triton X-100 overnight at $4^{\circ} \mathrm{C}$. Signal was visualized by a secondary antibody Alexa Fluor 488-conjugated donkey anti-mouse lgG (1:500, ab150105, Abcam plc.) or Alexa Fluor 594 AffiniPure donkey anti-mouse IgG (1:500, 715-585-150, Jackson ImmunoResearch) diluted in $0.5 \%$ PBS Triton $\mathrm{X}-100$ overnight at $4^{\circ} \mathrm{C}$. All sections were counterstained with 4',6-diamidino-2-phenylindole (DAPI; $1 \mu \mathrm{g} / \mathrm{ml}, 422801$, BioLegend), mounted with CC/Mount (Diagnostic BioSystems Inc.) and examined under a fluorescent microscope (MVX10, Olympus Corporation) or a laser scanning confocal microscope (FV1000, Olympus Corporation).

\section{Statistical analysis}

Statistical analyses were performed using jamovi (version 1.1.9, https://www.jamovi.org). Comparisons between more than two groups were analyzed by oneway or repeated measure two-way ANOVA followed by Tukey's HSD test for multiple comparisons. Pearson's correlation analysis determined the correlation between the body weight and presumptive travel distance on the running wheel. Statistical significance was defined as a value of $p<0.05$. Data are presented as mean \pm SEM.

\section{Software accessibility}

All the files for python program, bill of materials, microcontroller firmware, 3D-printed part, and printed circuit boards used in this study are available as Extended Data 1 and online (https://github.com/neurobio-hiroshima/WRAQ). 

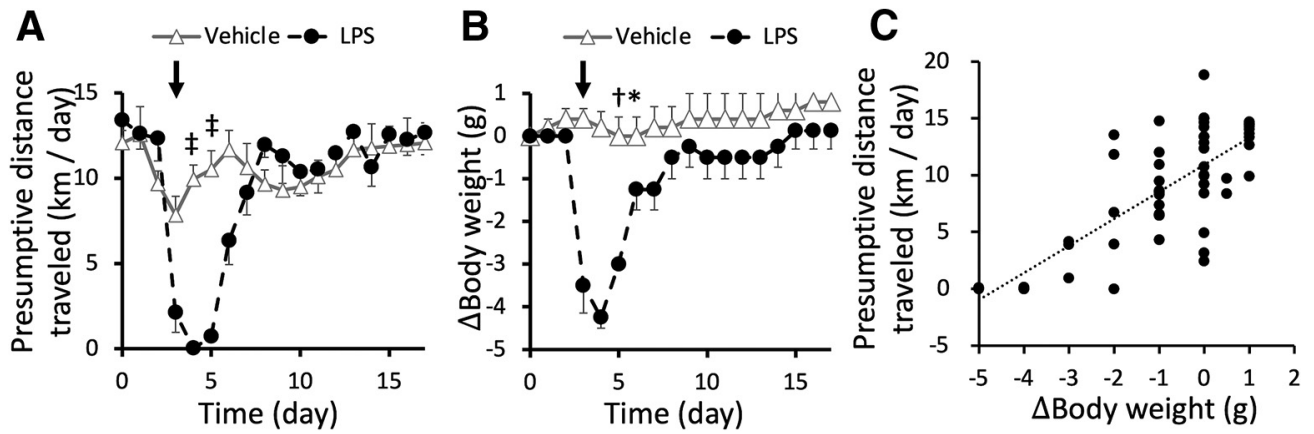

Figure 4. Transient suppression and recovery of wheel-running activity in a murine endotoxemia model. $\boldsymbol{A}, \boldsymbol{B}$, Line plots of daily wheel-running activity presented as the presumptive distance traveled $(\boldsymbol{A})$ and relative body weight change from baseline $(\boldsymbol{B})$ in mice with (filled circles with dashed lines) or without (triangles with solid lines) LPS treatment. Values are presented as mean \pm SEM. Arrows indicate the timing of LPS injection. C, Scatter plot of body weight change and presumptive distance traveled showing a positive correlation with statistical significance (Pearson's correlation coefficient $=0.745, p<0.001$ ). Contrasts are statistically significant differences between groups based on a repeated measure two-way ANOVA followed by Tukey's $p o s t$ hoc test; ${ }^{*} p<0.05$, $\dagger p<0.01, \neq p<0.001$.

\section{Results}

\section{Simultaneous recording of the wheel-running activity and illumination using WRAQ}

Mice in their home cage rotated the low-profile wheel of WRAQ already on the day of installation. The wheel revolutions gradually increased and plateaued around $7 \mathrm{~d}$ (Fig. $2 B, n=9$ mice). We determined whether WRAQ could simultaneously detect wheel-running activity and illumination intensity changes in the home cage per the scheduled illumination for LD cycles. Resistance changes in photoresistors resulted in a persistent decrease and increased voltage detected by WRAQ during the light and dark periods, respectively (Fig. 2C, top). Consistent with the nocturnal behavior of mice, wheel-running activity exhibited an abrupt increase and decreased following the onset and end of the dark period, respectively (Fig. 2C, bottom). Considering that WRAQ sampled data every $4 \mathrm{~s}$, these results indicate that WRAQ detected wheel-running activity of mice and the illumination intensity in their home cage with temporal precision.

\section{Long-term recording of the circadian rhythm in wheel- running activity under LD entrainment}

Next, we applied WRAQ to analyze the circadian rhythm of wheel-running activity in mice. Under a 12:12 h LD cycle, the number of wheel revolutions followed a diurnal rhythm with exclusive activity during the dark period (Fig. $3 A$ ). The active period started following the onset of the dark period. After a significant reduction of activity in the second half of the dark period, mice exhibited a shorter bout of wheel-running activity, which resulted in bimodal peaks of wheel-running activity, with distinct early and late-night activity bouts as reported previously (Pittendrigh and Daan, 1976).

Under altered schedules in light entrainment, particularly DD, WRAQ recorded the free-running in circadian rhythm with a shortening of the period. The resultant actogram exhibited a gradual advance of the onset of an active period on ZT (Fig. 3B). By contrast, LL led to variable changes in circadian rhythm, ranging from free-running with an elongated period (Fig. $3 C$ ) to an arrhythmic pattern (Fig. 3D). The LL condition also resulted in a significant reduction of the presumptive distance traveled compared with mice under LD or DD conditions (one-way ANOVA, $F_{(2,15.5)}=15.9$, Tukey's HSD test, $p<0.001, n=9$ mice for each group; Fig. 3E).

To measure the circadian rhythm in wheel-running activity, we subsequently calculated a periodogram using the ImageJ-based analysis software ActogramJ (Schmid et al., 2011). As an output of WRAQ, a comma-separated value file was imported into ActogramJ and analyzed using Lomb-Scargle methods. The results showed that the peak of the periodogram under LD was $\sim 24 \mathrm{~h}$ (mean peak value \pm SEM, $24.0 \pm 0.0844 \mathrm{~h}$ ). DD tended to shorten the period $(23.8 \pm 0.0533 \mathrm{~h})$, while LL elongated the period significantly $(25.9 \pm 0.2614 \mathrm{~h}$, oneway ANOVA, $F_{(2,7.52)}=28.3, p<0.001$, Tukey's HSD post hoc test, $p<0.001$ for both LL vs DD and LL vs LD) with free-running along the circadian rhythm (Fig. 3F).

These results indicate that WRAQ is a useful tool for quantifying wheel-running activity behavioral determinants and can be integrated into an open-source analysis.

\section{Alteration and recovery of the wheel-running activity in a murine endotoxemia model}

Systemic administration of LPS has been used as a model of endotoxemia, which induces systemic inflammation (Beutler, 2000). As a previous study showed a significant reduction of mice locomotor activity in an open field arena $24 \mathrm{~h}$ after systemic LPS injection (Giga et al., 2021), we applied WRAQ to determine mouse behavior before and after LPS to evaluate its applicability to murine disease models. As compared with the behavior before LPS injection, intraperitoneal administration of $2.5 \mathrm{mg} / \mathrm{kg}$ LPS significantly reduced voluntary wheel-running activity (repeated-measures ANOVA, group $\times$ time interaction, $p<0.001, F_{(17,153)}=8.95$; Tukey's post hoc test, $p<0.001$ for vehicle vs LPS at 


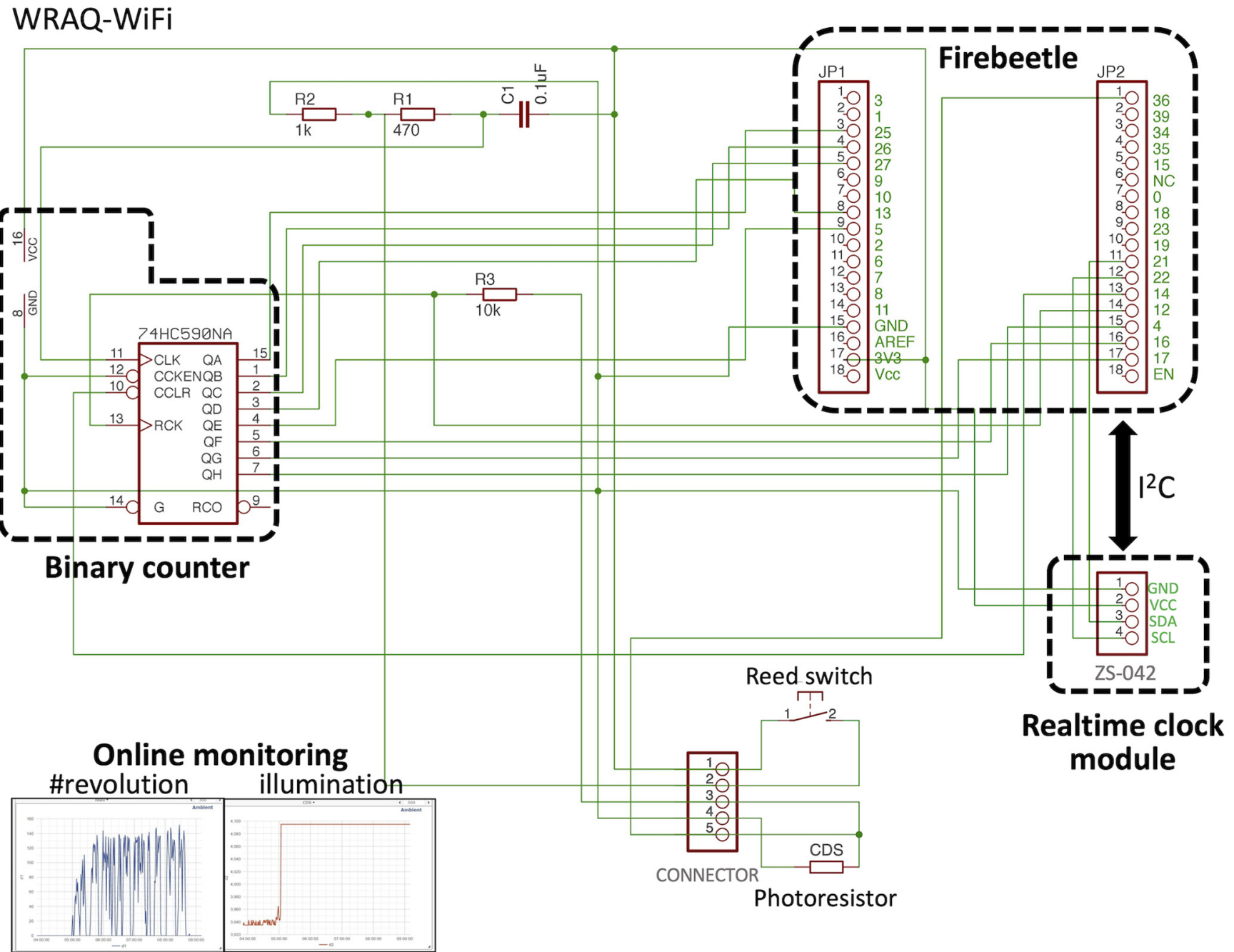

Figure 5. Wiring diagram for the WRAQ-WiFi. WRAQ-WiFi is based on ESP32 microcontroller implemented on a FireBeetle ESP32 board. The number of running wheel revolutions is measured by 8-bit counter 74HC590NA and transmitted to the cloud server and the timestamp (using real-time clock module) and illumination data in the home cage (based on change of photoresistor). Uploaded data are visualized on the online platform for real-time monitoring (panels at the lower-left corner). C, capacitor; CCLR; counter clear; CCKEN, counter clock enabled; CDS, cadmium sulfide photoresistor; CLK, clock input; G, ground; GND, ground; QA-QH, digital outputs from the binary counter; R, resistor; RCK, register clock; RES, reset; SDA, serial data line for I2C; SCL, serial clock line for $\mathrm{I}^{2} \mathrm{C}$; VCC, voltage common collector.

days 4 and $5, n=5$ and 7 for vehicle and LPS groups, respectively; Fig. 4A). This change was accompanied by a transient reduction of body weight (repeatedmeasures ANOVA, group $\times$ time interaction, $p<0.001$, $F_{(17,119)}=9.04$; Tukey's post hoc test, $p<0.001$ for vehicle vs LPS at days 5 and 6; Fig. $4 B$ ). Indeed, analysis revealed that the body weight change was positively correlated with wheel-running activity (Pearson's correlation coefficient $=0.745, p<0.001$; Fig. 4C), indicating that WRAQ detected a behavioral measure during endotoxemia inducing body weight loss. Intriguingly, long-term recording by WRAQ also unraveled a gradual recovery of wheel-running activity (Fig. 4A, black), further supporting the applicability of WRAQ to mouse models of diseases requiring longitudinal observation of long-lasting behaviors in the home cage.

\section{Chemogenetic activation of the SCN-SPZ shifts the onset of the active period under DD}

Based on our success in acquiring longitudinal data of wheel-running activity, we extended our system capability to enable online monitoring via data uploading to a cloud server. Implementation of this version of WRAQ as IoT (WRAQ-WiFi) integrates ESP32 microcontroller with WiFi capability (Fig. 5). The uploaded data remained available while WRAQ-WiFi was under-recording (Fig. 5).

During free-running along the circadian rhythm of mice under DD, we applied WRAQ-WiFi to manipulate neuronal activity at a specific CT. Studies showed that SCN-SPZ acted as a master clock and a region relaying the circadian information from the SCN to other brain regions, respectively (lbuka and Kawamura, 1975; Lu et al., 2001). We measured the wheel-running activity of a mouse targeted by AAV-hSyn-hM3D- 

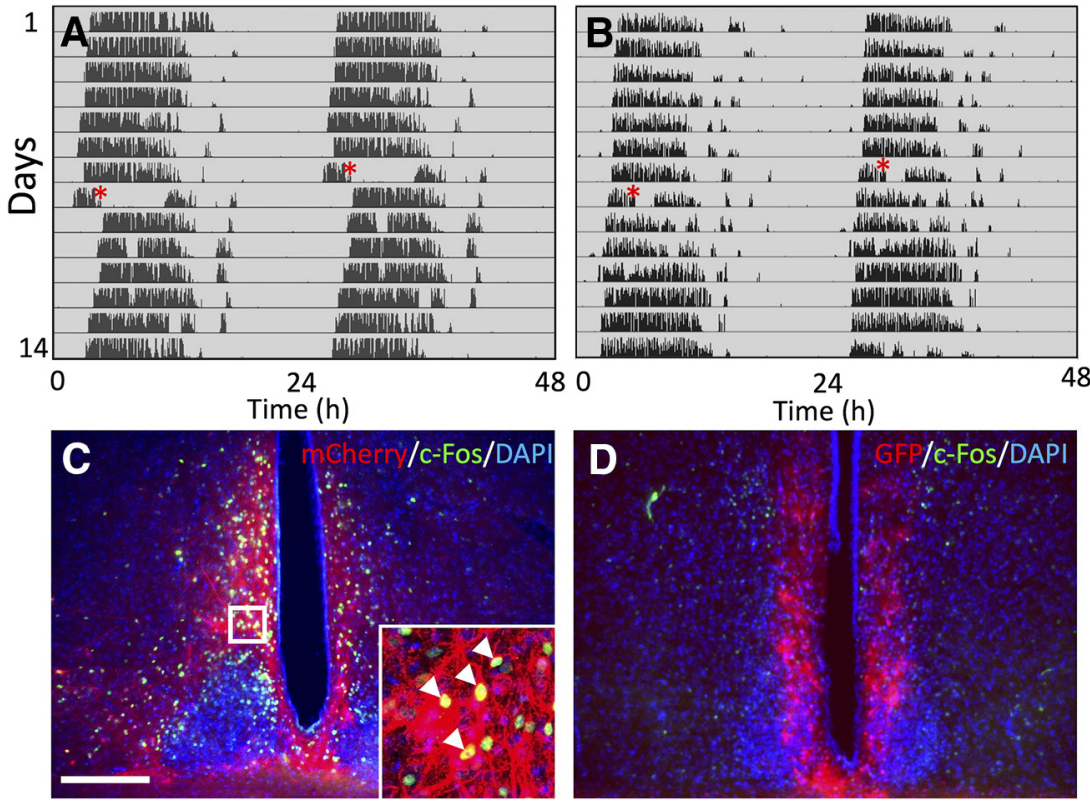

Figure 6. Temporarily specific neural activation patterns under the guidance of WRAQ-WiFi. $\boldsymbol{A}$, An actogram of mice receiving an injection of AAV8-Syn-hM3D-mCherry $(\boldsymbol{A})$ or AAV8-CAG-GFP $(\boldsymbol{B})$ to the SCN-SPZ under constant dark condition. On day 7, CNO was injected intraperitoneally at CT14, estimated based on the WRAQ-WiFi data available online (red asterisks in $\boldsymbol{A}, \boldsymbol{B}$ ). $\boldsymbol{C}, \boldsymbol{D}, \mathrm{Coronal}$ brain sections of the mouse used for behavioral analysis $(\boldsymbol{A}, \boldsymbol{B})$ showing the localization of mCherry (red in $\boldsymbol{C}$ ), GFP (pseudocolored red in $\boldsymbol{D}$ ), and c-Fos (green in $\boldsymbol{C}, \boldsymbol{D}$ ) in the SCN-SPZ. Independent of the behavioral recording in $\boldsymbol{A}, \boldsymbol{B}$, the mice brains were fixed $2 \mathrm{~h}$ after the CNO injection. Sections were counterstained with DAPI (blue in $\boldsymbol{C}, \boldsymbol{D}$ ). An inset is a magnified view of the boxed area in $\boldsymbol{C}$. White arrowheads indicate the cells co-expressing mCherry (red) and c-Fos. Scale bar in $\boldsymbol{C}$ (applies to $\boldsymbol{D}$ ): $200 \mu \mathrm{m}$.

mCherry to upregulate neuronal activity in the SCNSPZ on systemic administration of CNO (Fig. 6A,C, $n=5$ mice). We observed a significant increase of c-Fos-positive cells in the SCN-SPZ following intraperitoneal injection of CNO (Fig. 6C). Before CNO injection, the mouse exhibited free-running activity under DD (Fig. 6A). Under the guidance of WRAQ-WiFi, injection of CNO at CT14 (Fig. 6A, red asterisks) induced a significant shift in the onset of the active period lasting at least $7 \mathrm{~d}$ (Fig. 6A). In contrast, intraperitoneal injection of CNO to the mice expressing GFP in the SCN-SPZ did not induce any of these effects on the onset of active period (Fig. 6B) and C-Fos in SCNSPZ (Fig. 6D) significantly ( $n=5$ mice).

These data reveal that online monitoring with the WRAQ-WiFi system enables studies requiring a temporally specific genetic or pharmacological intervention.

\section{Discussion}

The present study demonstrated precise data acquisition of wheel-running activity along the circadian rhythm in mice within their home cage using the WRAQ system enabled by open-source hardware. The recorded data could be visualized offline and online when uploaded to the data visualization server in WRAQ-WiFi. Continuous monitoring of the wheel-running activity and circadian rhythm revealed altered activity and rhythms in mice exposed to systemic inflammation or a chemogenetic manipulation of a specific neuronal circuit. These results indicated that WRAQ is a novel tool that allows to explore the mechanisms underlying behaviors in murine disease models. We discuss below the utility of WRAQ and its limitation.

\section{Comparison with currently available technologies}

A general configuration of systems recording wheelrunning activity in rodents consists of microswitch and data acquisition board or interface with PC. Although communication between the running wheel with the switch and data acquisition parts can be wired or wireless, the presence of a data acquisition board or interface with PC can hamper their use in vivariums with limited space or high biosafety levels (Balcombe, 2006). WRAQ and WRAQ-WiFi store data on a built-in SD card or cloud server and work as a stand-alone device without requiring additional appendages, which allows their use in a wide variety of conditions generally encountered in mouse housing facilities. Because of its open-source nature, WRAQ-WiFi also has wide applicability for combination with other open-source loT platforms such as ThingsBoard.

The size of the WRAQ system (width 5 inches $\times$ depth 5 inches $\times$ height 3.5 inches) is smaller than representative low-profile running wheels (ENV-047, Med Associate Inc.; width 6.1 inches $\times$ depth 6 inches $\times$ height 4 inches) used widely in neuroscience studies with mouse models. Since the latter low-profile mouse running wheel was used successfully in standard "shoebox" style individually ventilated cages (e.g., model 9, Thoren Caging Systems with width 7.70 inches $\times$ depth 12.17 inches $\times$ height 5.875 
inches; Goh and Ladiges, 2015; Beeler and Burghardt, 2021), it is reasonable to think that our WRAQ fits a wide variety of cage systems.

Similar to other commercially available systems such as ClockLab (Actometrics, Co Ltd.), WRAQ/WRAQ-WiFi, built on open-source platform, is compatible with the recording of additional behaviors (e.g., general home cage activity, food and water consumption) and environmental data (e.g., temperature and humidity) for online monitoring using appropriate sensors.

\section{Limitation and future work}

Unlike data acquisition with high temporal resolution using commercially available systems, WRAQ and WRAQ-WiFi with 4- and 8-bit counters collect data on a binary counter every 4 and $60 \mathrm{~s}$, respectively, to save battery for long-term recording. The resulting temporal resolution in WRAQ is much lower than in other commercially available systems (e.g., ClockLab from Actometrics Co Ltd.). However, those intervals in WRAQ were set not to exceed the limit of binary counter based on a previous study showing that mice primarily run up to $105 \mathrm{~cm} / \mathrm{s}$ (Lemieux et al., 2016). However, it is unlikely that 4- and 60-s intervals are too short for long-term analysis over $24 \mathrm{~h}$.

In comparison with the wheel running activity recording system available commercially, in which the data analysis could be done on the same platform using single software (e.g., ClockLab), data analysis in WRAQ system has an additional step to convert the raw data using pipeline python program to match the data format between data acquisition system (Adalogger and Ambient loT server for WRAQ and WRAQ-WiFi, respectively) and analysis software (ActogramJ). This issue would be resolved by modifying the ActogramJ, which is also open-source software, to enable it to import raw data from WRAQ directly without conversion in the future.

Home cage activity can be measured using the general activity detected by motion sensors (Matikainen-Ankney et al., 2019). Since the running wheel significantly increases the home cage activity, WRAQ/WRAQ-WiFi is likely to affect the home cage activity per se. It would be interesting in the future to measure the general home cage activity detected by motion or capacitive sensors with or without functional WRAQ to address the influence of running wheel access on the home cage general activity.

\section{References}

Balcombe JP (2006) Laboratory environments and rodents' behavioural needs: a review. Lab Anim 40:217-235.

Beeler J, Burghardt N (2021) Activity-based anorexia for modeling vulnerability and resilience in mice. Bio Protoc 11:e4009.

Beutler B (2000) TIr4: central component of the sole mammalian LPS sensor. Curr Opin Immunol 12:20-26.

Franklin K, Paxinos G (2008) The mouse brain in stereotaxic coordinates. In: The spinal cord: a Christopher and Dana Reeve Foundation text and atlas, Ed 3. New York: Elsevier.

Giga H, Ji B, Kikutani K, Fukuda S, Kitajima T, Katsumata S, Matsumata M, Suhara T, Yamawaki S, Shime N, Hosokawa K, Aizawa H (2021) Pharmacological and genetic inhibition of translocator protein $18 \mathrm{kDa}$ ameliorated neuroinflammation in murine endotoxemia model. Shock 56:142-149.

Goh J, Ladiges W (2015) Voluntary wheel running in mice. Curr Protoc Mouse Biol 5:283-290.

Ibuka N, Kawamura H (1975) Loss of circadian rhythm in sleepwakefulness cycle in the rat by suprachiasmatic nucleus lesions. Brain Res 96:76-81.

Lemieux M, Josset N, Roussel M, Couraud S, Bretzner F (2016) Speed-dependent modulation of the locomotor behavior in adult mice reveals attractor and transitional gaits. Front Neurosci 10:42.

Lu J, Zhang YH, Chou TC, Gaus SE, Elmquist JK, Shiromani P, Saper CB (2001) Contrasting effects of ibotenate lesions of the paraventricular nucleus and subparaventricular zone on sleepwake cycle and temperature regulation. J Neurosci 21:4864-4874.

Matikainen-Ankney BA, Garmendia-Cedillos M, Ali M, Krynitsky J, Salem G, Miyazaki NL, Pohida T, Kravitz AV (2019) Rodent activity detector (RAD), an open source device for measuring activity in rodent home cages. eNeuro 6 6:ENEURO.0160-19.2019.

Novak CM, Burghardt PR, Levine JA (2012) The use of a running wheel to measure activity in rodents: relationship to energy balance, general activity, and reward. Neurosci Biobehav Rev 36:1001-1014.

Pittendrigh CS, Daan S (1976) A functional analysis of circadian pacemakers in nocturnal rodents. J Comp Physiol A Neuroethol Sens Neural Behav Physiol 106:333-355.

Schmid B, Helfrich-Förster C, Yoshii T (2011) A new ImageJ plug-in "ActogramJ" for chronobiological analyses. J Biol Rhythms 26:464-467.

Schneider CA, Rasband WS, Eliceiri KW (2012) NIH Image to ImageJ: 25 years of image analysis. Nat Methods 9:671-675.

Siepka SM, Takahashi JS (2005) Methods to record circadian rhythm wheel running activity in mice. Methods Enzymol 393:230-239.

Takahashi JS (2017) Transcriptional architecture of the mammalian circadian clock. Nat Rev Genet 18:164-179. 